\title{
matters \\ Postsynaptic Rab GTPases and Exocyst: a screen at the Drosophila neuromuscular junction
}

\author{
Cátia F Patrício-Rodrigues, Rita O Teodoro \\ CEDOC, Nova Medical School, Universidade Nova de Lisboa
}

\author{
$\nabla$ Correspondence \\ rita.teodoro@nms.unl.pt \\ 8 Disciplines \\ Cell Biology \\ Q Keywords \\ Rab GTPases \\ Drosophila Melanogaster \\ Exocyst \\ Neuromuscular Junction \\ Ral GTPase

\section{Type of Observation Standalone \\ S. Type of Link} \\ Standard Data \\ (1) Submitted Jun 15, 2018 \\ (1) Published Jul 24, 2018
}

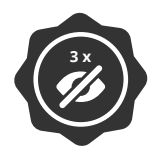

Triple Blind Peer Review The handling editor, the reviewers, and the authors are all blinded during the review process.

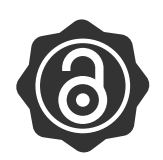

Full Open Access Supported by the Velux Foundation, the University of Zurich, and the EPFL School of Life Sciences.

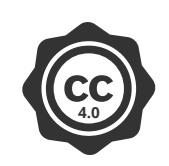

Creative Commons 4.0 This observation is distributed under the terms of the Creative Commons Attribution 4.0 International License.

\begin{abstract}
Neuronal morphology is established during development but can subsequently be modified by synaptic activity, a process known as structural plasticity. Postsynaptic compartments, such as dendritic spines or the postsynaptic membrane of the Drosophila neuromuscular junction (NMJ) (called the subsynaptic reticulum or SSR), are highly dynamic elements that grow during development, and that are subject to this type of plasticity. While it is known that the shape of postsynaptic structures is tightly coupled to synaptic function, the factors that govern the morphology and its relationship with functional plasticity are still elusive. Ral GTPase has been shown to regulate the expansion of postsynaptic membranes during development and in response to synaptic activity via the Exocyst, an octameric tethering complex conserved from yeast to human. Postsynaptic activation of Ral at the Drosophila NMJ induces Exocyst recruitment to the synapse, resulting in membrane addition and SSR growth. However, how this type of remodeling is actually achieved, remains to be determined. Given the known role of Rab GTPases in polarized delivery of vesicles, we expect that a subset of these will be required for Exocyst-dependent SSR growth. Here, by systematically evaluating the localization of the Exocyst subunit Sec5 after postsynaptic activation of each Rab GTPase, we concluded that no single Rab is able to induce Sec5 recruitment to the NMJ to same extent as Ral GTPase. This result may indicate that activation of Ral at the Drosophila NMJ is necessary to initiate the signaling cascade that controls SSR size. In addition, we describe the cellular distribution of postsynaptic active Rab GTPases, and identify putative candidates whose distribution and relationship with the Exocyst may indicate a Rab/Exocyst-dependent role in muscle and/or postsynaptic development. Altogether, this study contributes to untangle the vesicle trafficking pathway(s) that regulate SSR growth at the Drosophila NMJ.
\end{abstract}

\section{Introduction}

The correct wiring of the nervous system is required for functions such as learning and memory, locomotion, or perception. Regulation of neuronal morphology is critical for function and, to achieve the correct structure, constant and orchestrated intracellular trafficking is required [1]. The Ral/Exocyst pathway has been shown to be necessary for postsynaptic development and activity-dependent membrane growth at the Drosophila NMJ [2] [3], but how growth is achieved remains elusive. Central to the mechanism of synaptic plasticity discussed above, is the activation of the Exocyst complex. The Exocyst is an effector to many GTPases, including Ral and Rab GTPases. Rab GTPases are a class of proteins that confer membrane identity to intracellular compartments, and that are capable of coordinating and organizing downstream effectors [4] [5]. Similarly, the Exocyst complex is a tethering complex that, by being able to receive regulatory information from different pathways, can serve as a hub to precisely regulate where and when vesicles fuse [6]. Because of this central role in directing trafficking, knowing the regulation of Rabs and Exocyst will be key to understanding neuronal organization and plasticity. Dissecting the signaling cascade regulated by Ral activation will complete our knowledge on the pathway through which Ral/Exocyst regulate SSR growth.

\section{Objective}

Our broad objective is to find new regulators of postsynaptic membrane size. Because growth requires membrane addition and Rab GTPases and the Exocyst complex are master regulators of cellular traffic, we want to uncover which Rab GTPases are required for postsynaptic membrane growth at the Drosophila NMJ, and whether they colocalize with the Exocyst. Specifically, our objectives are: 
1. Determine the localization of active Rabs when expressed in the muscle.

2. Test if expression of each active RabCA can induce Exocyst recruitment to the post-

synapse.

3. Establish whether there is colocalization of the Exocyst with each active Rab.

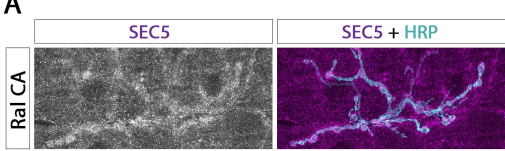

C
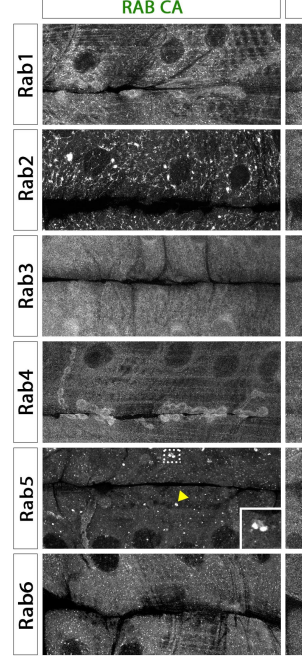

家
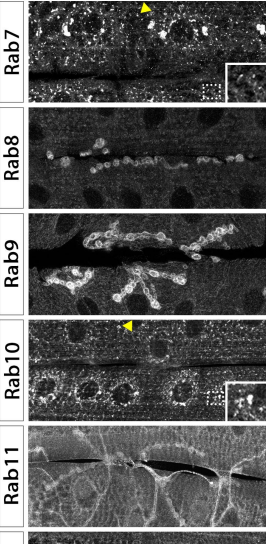

$\frac{7}{8}$

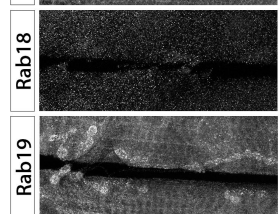

\begin{tabular}{|l|l|l|l|l|l|l|l|l|l|l|l|l|l|l|} 
Rabs CA & Rab1 & Rab2 & Rab3 & Rab4 & Rab5 & Rab6 & Rab7 & Rab8 & Rab9 & Rab10 & Rab11 & Rab14 & Rab18 & Rab19 \\
\hline
\end{tabular}

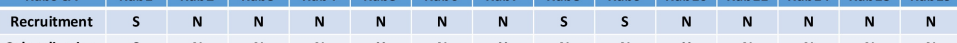

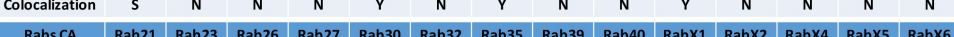

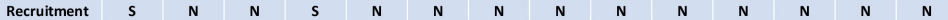

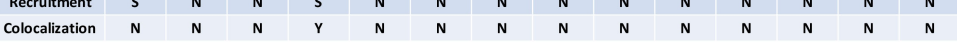

MERGE + HRP
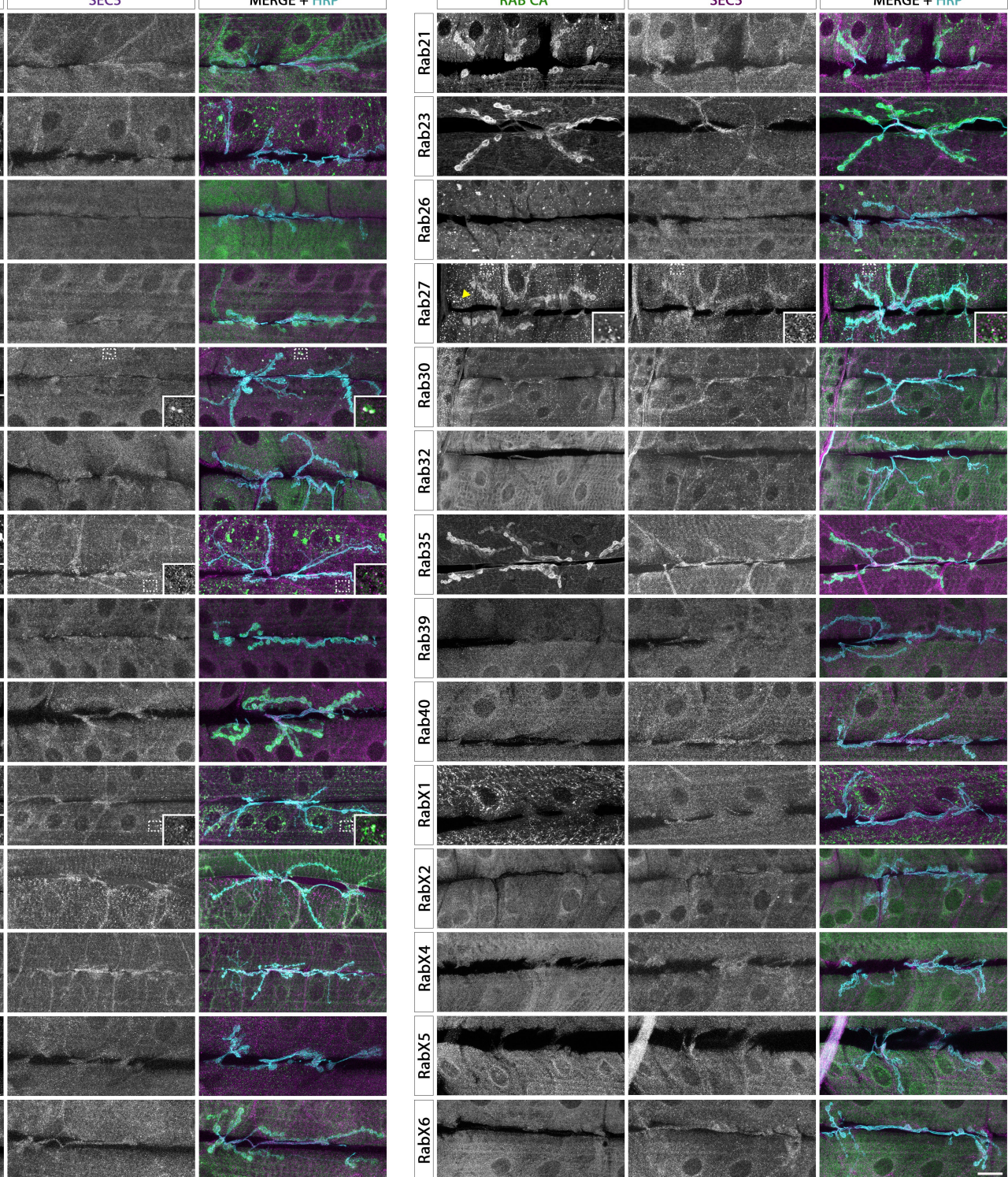

Figure Legend

Figure 1. A screen for Sec5 recruitment to the NMJ upon muscle activation of Rab GTPases.

(A) Expression of Ral GTPase in the constitutively active form in the muscle (MHCGS;RalCA) induces Sec5 (grey) recruitment to the NMJ. Neuronal membrane is marked with anti-HRP (cyan).

(B) Summary table of muscle RabCA expression: analysis of Sec5 recruitment (top row) 
and Rab and Sec5 colocalization (bottom row). Images of the summary table are represented in panel C. Table key: N-No, Y-Yes, S-Some.

(C) Muscle expression of RabCAs (MHC-GS; UAS-RabCA-YFP). Each row per column is a representative example of each individual RabCA. We conclude that muscle RabCA epression does not mimic muscle RalCA (Fig. 1A). RabCA-YFP is labelled with anti-GFP antibody (green), Sec5 is labelled with anti-Sec5 (magenta) and the neuronal membrane is marked with anti-HRP (cyan). When localization between RabCA and Sec5 was observed in single sections, a zoomed image is shown in the bottom right corner. Dashed square shows region of interest. Yellow arrowheads show bright GFP puncta that do not colocalize with Sec5, indicating that colocalization is not due to channel bleed-through. Scale Bar $20 \mathrm{~mm}$.

\section{Results \& Discussion}

The genetic pathway(s) that regulates SSR growth remains poorly understood. Normally, the Exocyst is ubiquitously distributed, but expression of RalCA in the muscle induces a strong recruitment of Sec5 to the NMJ (Fig. 1A and 2). To explore the role of Rab GTPases in postsynaptic development and function, we used the UAS-Gal4 system to express a collection of constitutively active YFP-tagged Rab GTPases (RabCA) in the muscle of Drosophila larvae [7]. For this, we used MHC-GeneSwitch-Gal 4 (MHC-GS) and crossed it with each individual RabCA. We labeled each Rab with YFP, the Exocyst with Sec 5 and the motor neuron membrane using HRP. Expression of each active Rab in the muscle allows the assessment of: 1) localization, 2) induction of Sec5 recruitment to the NMJ (as a proxy for vesicle delivery to the SSR), and 3) Rab colocalization with the Exocyst component Sec5. The analysis of these 3 points will contribute to the understanding of the distribution of Rab GTPases in the muscle and to assess their putative interactions with the Exocyst complex. The results obtained are summarized in figure $1 \mathrm{~B}-\mathrm{C}$.

If a given Rab is involved in SSR growth, one would expect that it localizes to the NMJ or that it regulates a membrane flow that favors exocytosis and membrane addition at the NMJ. It has been shown that about $50 \%$ of all Rabs are endogenously enriched or exclusively expressed in neurons, with most being enriched presynaptically, and Rab2 1 and 23 mostly with postsynaptic expression [8]. In supplement, we show the endogenous distribution of a pre- Rab tagged with YFP in the endogenous promoter (Rab3), to exemplify pre- versus postsynaptic localization of Rabs (Fig. 1), relative to the neuronal membrane [9]. Upon postsynaptic expression of Rabs, our data revealed that Rab 4, 8, 9, 19, 21, 23, 27 and 35 are clearly enriched at the NMJ, while Rab1, 5, 11 and 14 show some NMJ localization, despite less pronounced (Fig. ${ }_{1} \mathrm{C}$ ). Some Rabs are enriched at the perinuclear area (Rab1, 2, 10, 30, 32, X1, X2 and $\left.\mathrm{X}_{5}\right)$ and others are uniformly distributed throughout the muscle (Fig. $1 \mathrm{C}$ ). The presence of Rabs at the NMJ suggests that they may play a role at this specialized postsynaptic membrane. However, whether they participate in SSR growth or in other processes related to postsynaptic function or development (namely glutamate receptor endo or exocytosis) cannot be concluded from this data.

One pathway that has been shown to regulate SSR growth is Ral GTPase via the Exocyst complex. This pathway is required for developmental and activity-dependent SSR growth, and to promote Sec5 recruitment to the NMJ upon muscle RalCA overexpression (Fig. 1A). If muscle RalCA induces Sec5 recruitment to the NMJ (Fig. 1A), we hypothesized that we could screen for Rabs required for SSR growth by expressing each RabCA in the muscle, and assessing whether this manipulation changes Sec5 distribution. To our surprise, no single active Rab is able to induce Sec 5 recruitment to the NMJ to the same extent as RalCA (compare Fig. 1A with C). Despite this, we saw a small, but reproducible recruitment of Sec5 upon activated Rab 1, 8, 9, 14, 21 or 27 expression, with Rab27 having the strongest effect. This effect was observed in all larvae analyzed. Interestingly, Rab27 has been shown to be involved in regulated and constitutive secretion pathways, where Rab27 effectors are required for the transport and docking of vesicles, prior to their secretion to the membrane [10]. In fact, the Myosin Va and Rab27 interacting protein MyRIP, has been shown to bind to the Exocyst complex, establishing a ternary complex with Rab27 and MyoVa that regulates the transport and targeting of 
secretory granules to the plasma membrane [11]. Given the mechanistic similarities, it is tempting to speculate that a similar mechanism could operate for Exocyst-dependent SSR growth (possibly during development and/or in response to activity). Also of interest is the identification of Rab1 as a candidate: Rab1 has been identified in a screen for dendritic growth of Drosophila sensory neurons [12]. Rab1 is a critical regulator of ER-Golgi transport via COPII vesicles, being therefore in a key position to regulate the flux of secretory vesicles that can contribute to SSR growth. Other candidate- Rab8, has been shown to interact with the Exocyst and RalGTPase in the regulation of membrane addition during furrow formation by directing vesicles to the place of furrow ingression [13] [14]. Rab14 is upstream of Rab1o in the regulation of Glut4 to the membrane [15], a process also dependent on Ral GTPase [16]. In our experiments, we do not see Sec5 recruitment after Rab1oCA expression, but we do see colocalization between Sec5 and Rab1o (Fig. 1). Additionally, Rab1o is also required for membrane secretion during organ development [17] and for dendritic growth in C. elegans [18], which raises the possibility that Rab14, Rab1o and Exocyst could potentially be involved in directing vesicles to membranes marked by Ral, contributing this way to the regulation of SSR size. Rab9 and Rab21, even though being present at the NMJ and inducing some Sec5 recruitment, have been related with endocytic processes and are less obviously involved in SSR growth [19] [20]. In spite of having identified these candidates, it is clear that no Rab can fully mimic the effects of RalCA. Ral is localized at the plasma membrane where, when activated, serves to localize the Exocyst and direct secretory vesicles to the membrane to induce SSR growth [2]. Given the weak phenotypes observed with RabCA expression, a possible explanation is that Ral is the main- perhaps limiting, factor present at the plasma membrane and that without activating this small GTPase, it is not possible to promote the recruitment of Exocyst vesicles to the NMJ, and concomitant SSR growth.

Finally, we analyzed colocalization between the Rabs and Sec5 as a proxy for common function. Despite both Rabs and Sec5 having a widespread distribution, the extent of colocalizing puncta is small. We analyzed colocalization of Sec 5 and each Rab, in single sections, and observed colocalization with Rab5, 7, 10 and 27. These Rabs label respectively, early endosomes, late endosomes/lysosomes, recycling compartments (Rab1o), and Rab27 labeling some specialized secretory vesicles and exosomes. Altogether, in this study, we establish the localization of active Rabs when expressed in the postsynaptic muscle, and identified Rab candidates that may interact with the Exocyst in their active state, suggesting that they may be functionally related. We also concluded that activation of Rabs in the muscle is not sufficient to promote Sec5 recruitment to the NMJ, akin to what occurs with Ral.

\section{Conclusions}

Activation of Ral GTPase leads to Exocyst recruitment to the NMJ and SSR growth [2] [3]. Here, we report that no single active Rab is able to recruit the Exocyst to the NMJ to the same extent as Ral. Albeit not as strong, we found that Rab1, 8, 9, 14 and 27 may contribute to Ral/Exocyst-dependent SSR growth, but additional studies will be required to assess this contribution. In addition, we show that active Rab5, 7, 10 and 27 colocalize with the Exocyst component Sec5, suggesting that they may play some function in this tissue (muscle/NMJ). We suggest that Ral likely functions at the NMJ as a master regulator of SSR growth, whose activation is necessary for Exocyst-dependent trafficking of secretory vesicles.

\section{Limitations}

Overexpression of GTP-locked Rab GTPases prevents the cycling between GDP and GTP potentially leading to altered function. This requirement to cycle could also explain why no RabCA is able to recruit Sec5 as RalCA does. However, given that Ral is also a GTPase from the same superfamily, this problem should also affect Ral: but this issue remains a formal possibility. In addition, this approach can result in the mistargeting of RabCA to membranes that are not in their normal place of action. While these issues are potential problems, this type of strategy has successfully been used in 
the past, and is informative. Furthermore, we have no indication of gross abnormalities upon RabCA over-expression in the muscle. We were able to identify candidates, which can now be further studied. Another limitation of this study is that it is mostly qualitative, preventing the identification of subtle effects induced by RabCA over-expression. A deeper analysis can be done if each Rab is studied in detail.

\section{Additional Information}

\section{Methods and Supplementary Material}

Please see https://sciencematters.io/articles/201807000005.

\section{Funding Statement}

This work was supported by H2020-GA661543-Neuronal Trafficking (ROT), IF/oo392/2013/CP1192/CTooo2, FCT - Portugal (ROT) and by UID/Multi/o4462/2013 from iNOVA4 4 Health (co-fundend by FCT-FEDER-PT2020).

\section{Acknowledgements}

We would like to thank João Mauricio for initial RabCA stainings. We thank Dr. Telmo Pereira, from the Microscopy Facility in the Chronic Diseases Research Center (CEDOC) of the Nova Medical School.

Ethics Statement

Not Applicable.

\section{Citations}

[1] Pico Caroni, Flavio Donato, and Dominique Muller. "Structural plasticity upon learning: regulation and functions". In: Nature Reviews Neuroscience 13.7 (2012), pp. 478-490. DOI: 10. $1038 /$ nrn3258. URL: https://doi.org/10.1038/nrn3258.

[2] Rita O Teodoro et al. "Ral mediates activity-dependent growth of postsynaptic membranes via recruitment of the exocyst". In: The EMBO fournal 32.14 (2013), pp. 2039-2055. DOI: 10.1038/emboj. 2013.147. URL: https://doi.org/10.1038/emboj.2013.147.

[3] GaYoung Lee and Thomas L Schwarz. "Filamin, a synaptic organizer in Drosophila, determines glutamate receptor composition and membrane growth". In: eLife 5 (2016), e19991. DoI: $10.7554 /$ elife. 19991. URL: https://doi.org/10.7554/elife. 19991.

[4] Francis A. Barr. "Rab GTPases and membrane identity: Causal or inconsequential?" In: The fournal of Cell Biology 202.2 (2013), pp. 191-199. DOI: $10.1083 / \mathrm{jcb} .201306010$. URL: https://doi.org/10.1083/jcb.201306010.

[5] Ferdi Ridvan Kiral et al. "Rab GTPases and Membrane Trafficking in Neurodegeneration". In: Current Biology 28.8 (2018), R471-R486. DOI: $10.1016 / \mathrm{j}$. cub . 2018.02 .010 . URL: https://doi.org/10.1016/j.cub.2018.02.010.

[6] Magdalena Martin-Urdiroz et al. "The Exocyst Complex in Health and Disease". In: Frontiers in Cell and Developmental Biology 4 (2016), p. 24. DOI: $10.3389 /$ fcell .2016 .00024 . URL: https://doi.org/10.3389/fcell.2016.00024.

[7] Jun Zhang et al. "Thirty-One Flavors of Drosophila Rab Proteins" In: Genetics 176.2 (2006), pp. 1307-1322. DoI: 10.1534/genetics.106.066761. urL: https : //doi.org/10.1534/genetics.106.066761.
[8] Chih-Chiang Chan et al. "Systematic Discovery of Rab GTPases with Synaptic Functions in Drosophila”. In: Current Biology 21.20 (2011), pp. 1704-1715. DOI: 10.1016/j.cub.2011.08.058. URL: https://doi.org/10.1016/j.cub.2011.08.058.

[9] Sebastian Dunst et al. "Endogenously Tagged Rab Proteins: A Resource to Study Membrane Trafficking in Drosophila". In: Developmental Cell 33.3 (2015), pp. 351-365. Dor: $10.1016 / j$. devcel.2015.03.022. URL: https : //doi.org/10.1016/j.devce1.2015.03.022.

[10] Mitsunori Fukuda. "Rab27 Effectors, Pleiotropic Regulators in Secretory Pathways". In: Traffic 14.9 (2013), pp. 949-963. DoI: $10.1111 /$ tra. 12083 . URL https://doi.org/10.1111/tra.12083.

[11] Sébastien Huet et al. "Myrip Couples the Capture of Secretory Granules by the Actin-Rich Cell Cortex and Their Attachment to the Plasma Membrane". In: Fournal of Neuroscience 32.7 (2012), pp. 2564-2577. DOI: 10.1523/jneurosci.2724-11.2012. uRL: https : //doi.org/10.1523/jneurosci.2724-11.2012.

[12] Bing Ye et al. "Growing Dendrites and Axons Differ in Their Reliance on the Secretory Pathway". In: Cell 130.4 (2007), pp. 717-729. DOI: $10.1016 / \mathrm{j}$. cell .2007 .06.032. URL: https:

//doi.org/10.1016/j.cel1.2007.06.032.

[13] Ryan M. Holly et al. "A rapid, membrane-dependent pathway directs furrow formation through RalA in the early Drosophila embryo". In: Development 142.13 (2015), pp. 2316-2328. DOI: $10.1242 / \mathrm{dev} .120998$. URL: https://doi.org/10.1242/dev.120998

[14] Lauren M. Mavor et al. "Rab8 directs furrow ingression and membrane addition during epithelial formation in Drosophila melanogaster". In: Development 143.5 (2016), pp. 892-903. DOI: $10.1242 / \mathrm{dev} .128876$. URL: https://doi.org/10.1242/dev.128876. 
[15] L. Amanda Sadacca et al. "Specialized sorting of GLUT4 and its recruitment to the cell surface are independently regulated by distinct Rabs". In: Molecular Biology of the Cell 24.16 (2013), pp. 2544-2557. DOI: $10.1091 / \mathrm{mbc}$. e13-02-0103. URL: https://doi.org/10.1091/mbc.e13-02-0103.

[16] Sheelarani Karunanithi et al. "A Rab1o:RalA G protein cascade regulates insulin-stimulated glucose uptake in adipocytes”. In: Molecular Biology of the Cell 25.19 (2014), pp. 3059-3069. DOI: 10.1091/mbc. e14-06-1060. URL: https://doi.org/10.1091/mbc. e14-06-1060.

[17] David W. Lerner et al. “A Rab1o-Dependent Mechanism for Polarized Basement Membrane Secretion during Organ Morphogenesis". In: Developmental Cell 24.2 (2013), pp. 159-168. DoI: $10.1016 / \mathrm{j}$.devcel . 2012.12.005. uRL: https: //doi.org/10.1016/j.devce1.2012.12.005.

[18] Wei Zou et al. "RAB-10-Dependent Membrane Transport Is Required for Dendrite Arborization”. In: PLOS Genetics 11.9 (2015), e1005484. DOI:

10.1371/journal.pgen. 1005484 . urL: https : //doi.org/10.1371/journal.pgen. 1005484

[19] Steve Jean et al. "Sbf/MTMR13 coordinates PI(3)P and Rab21 regulation in endocytic control of cellular remodeling”. In: Molecular Biology of the Cell 23.14 (2012), pp. 2723-2740. DOI
$10.1091 / \mathrm{mbc}$ e $12-05-0375$. URL:

https://doi.org/10.1091/mbc.e12-05-0375.

[20] Bo Dong et al. "Rab9 and retromer regulate retrograde trafficking of luminal protein required for epithelial tube length control”. In: Nature Communications 4.1 (2013), p. 1358. DOI:

10.1038/ncomms2347. URL:

https://doi.org/10.1038/ncomms 2347

[21] Thomas Osterwalder et al. "A conditional tissue-specific transgene expression system using inducible GAL4”. In: Proceedings of the National Academy of Sciences 98.22 (2001), pp. 12596-12601. DOI: 10 . 1073/pnas . 221303298 . URL https://doi.org/10.1073/pnas. 221303298.

[22] Jonathan R. Brent, Kristen M. Werner, and Brian D. McCabe. "Drosophila Larval NMJ Dissection". In: fournal of Visualized Experiments 24.24 (2009), e1107. DOI: 10 . 3791/1107. URL: https://doi.org/10.3791/1107.

[23] Mala Murthy et al. "Mutations in the Exocyst Component Sec5 Disrupt Neuronal Membrane Traffic, but Neurotransmitter Release Persists". In: Neuron 37.3 (2003), pp. 433-447. DOI: 10.1016/s0896-6273(03)00031-X. uRL: https : //doi.org/10.1016/s0896-6273(03)00031-x. 